This item was submitted to Loughborough's Research Repository by the author.

Items in Figshare are protected by copyright, with all rights reserved, unless otherwise indicated.

\title{
A remote approach to measure blood perfusion from the human face
}

PLEASE CITE THE PUBLISHED VERSION

http://dx.doi.org/10.1117/12.807354

PUBLISHER

(c) SPIE

VERSION

VoR (Version of Record)

\section{PUBLISHER STATEMENT}

This work is made available according to the conditions of the Creative Commons Attribution-NonCommercialNoDerivatives 4.0 International (CC BY-NC-ND 4.0) licence. Full details of this licence are available at: https://creativecommons.org/licenses/by-nc-nd/4.0/

\section{LICENCE}

CC BY-NC-ND 4.0

\section{REPOSITORY RECORD}

Zheng, Jia, Sijung Hu, Vicente Azorin-Peris, Angelos S. Echiadis, Ping Shi, and V.A. Chouliaras. 2019. "A Remote Approach to Measure Blood Perfusion from the Human Face". figshare.

https://hdl.handle.net/2134/22204. 


\title{
A remote approach to measure blood perfusion from the human face
}

\author{
Jia Zheng, Sijung Hu*, Angelos S. Echiadis, Vince Azorin-Peris, Ping Shi, Vasilios Chouliaras \\ Department of Electronic and Electrical Engineering, Loughborough University, Ashby Road, \\ Loughborough, Leicestershire, LE11 3TU, UK
}

\begin{abstract}
A CMOS camera-based imaging photoplethysmography (PPG) system has been previously demonstrated for the contactless measurement of skin blood perfusion over a wide tissue area. An improved system with a more sensitive CCD camera and a multi-wavelength RCLED ring light source was developed to measure blood perfusion from the human face. The signals acquired by the PPG imaging system were compared to signals captured concurrently from a conventional PPG finger probe. Experimental results from eight subjects demonstrate that the camera-based PPG imaging technique is able to measure pulse rate and blood perfusion.
\end{abstract}

Keywords: photoplethysmography, remote, dual-wavelength illumination, resonant cavity light emitting diode, pulse rate.

\section{INTRODUCTION}

The application of optical technology in medicine and biology has a long and distinguished history. Since the 18th century, the microscope has been an indispensable tool of biologists ${ }^{[1]}$. Pulse oximetry one of the most popular applications of Photoplethysmography (PPG) which was devised in the 1930 ' $\mathrm{s}^{[2]}$, measures oxygen saturation in blood by taking the ratio of light absorbed at two different wavelengths. With the invention of the laser in the early 1960's, physicians gained a new surgical instrument ${ }^{[3]}$. The past few years have seen considerable advances in understanding light propagation in tissue, resulting in the development of tomographic techniques, shifting from conventional medical optical methods into imaging and tomography.

PPG imaging is one of the emerging medical imaging modalities. The purpose of any new medical technology is to help clinicians reach a decision fast and accurately, to relieve patients from pain and to reduce morbidity and mortality. PPG imaging can provide information about both the tissue structure and the perfusion state of the tissue. It has great potential towards remote sensing, reducing the physical restrictions and cabling associated with patient monitoring. It can also provide benefits in removing potentially injurious wires from magnetic resonance imaging (MRI) machines ${ }^{[4]}$ and avoiding the time-consuming scanning of laser Doppler perfusion imaging (LDPI) ${ }^{[5]}$.

The feasibility of the camera-based PPG system has been previously demonstrated by means of blood perfusion visualization $^{[6,7]}$. A previous research report ${ }^{[8]}$ presents a remote reflection-mode CMOS camera-based system with a ring light of similar wavelengths $(660$ and $840 \mathrm{~nm})$ capturing "heart cycle-related" pulsatile variations. However, simultaneous two-wavelength image acquisition was not achieved, and the arterial pulsation signal was too weak to be recognized. Other previous research ${ }^{[9]}$ shows a camera-based system capable of capturing PPG waveforms at two different wavelengths simultaneously using a matrix of LEDs for illumination. This paper presents a non-contact camerabased system with a two-wavelength RCLED (resonant cavity LED) ring light source, capable of capturing clear PPG waveforms at two different wavelengths $(650 \mathrm{~nm}$ and $870 \mathrm{~nm})$ from the human face. This study compares the performance of PPG imaging with respect to conventional contact PPG, and the results show the capability of the system to obtain quality PPG waveforms for monitoring the pulse rate from the human face.

\footnotetext{
* S.Hu@lboro.ac.uk; phone +44 1509227058
} 


\section{METHODS}

\subsection{Instrumentation}

The PPG imaging system as shown in Fig. 1 employs a CCD camera (C10000-201, Hamamatsu, Japan) with a maximum resolution of $2048 \times 128$ pixels (pixel size $12 \mu \mathrm{m}^{2}$ ). The pixels are encoded using 12 bits, providing a high digitization resolution (4096 intensity levels). The camera was connected to a personal computer (PC) via a Camera Link ${ }^{\circledR}$ frame grabber PCI card (Inspecta-5, Mikrotron $\mathrm{GmbH}$ ) and the streamed frames were recorded in a custom-designed LabView virtual instrument (National Instruments Corp., USA). An industry-standard F-Mount zoom lens (focal length: 50mm, $\mathrm{f} / 1.8 \mathrm{D}$, AF Nikkor, Japan) was attached to the camera and depending upon the settings, it was possible to observe arbitrary areas of the skin surface, from a few square millimeters to several square centimeters. The camera was mounted on an optical bench with the lens positioned approximately $13 \mathrm{~cm}$ away from the human face under investigation.

A custom built dual-wavelength RCLED ring light source $\left(\lambda_{1}: 650 \mathrm{~nm}, \lambda_{2}: 870 \mathrm{~nm}\right)$ with a parabolic reflector (DIA: $18 \mathrm{~cm}$, O.L: $6.5 \mathrm{~cm}$ ) to provide collimated and uniform light, was mounted around the camera lens. The ring light consisted of 20 RCLEDs-10 with a peak wavelength of $650 \mathrm{~nm}$ (TRC650SMD0603, WelTek Co. Ltd., Taiwan), each emitting $1.0 \mathrm{~mW}$ power at a forward current $\left(\mathrm{I}_{\mathrm{f}}\right)$ of $20 \mathrm{~mA}$, and 10 with a peak wavelength of $870 \mathrm{~nm}$ (TRC870SMD0603, WelTek Co. Ltd., Taiwan) emitting $1.3 \mathrm{~mW}$ power at $\mathrm{I}_{\mathrm{f}}=20 \mathrm{~mA}$ individually. The arrangement of the RCLEDs is shown in the lower inset of Fig. 1. A control circuit with a microcontroller (PIC16F876A, MicroChip Inc., USA) alternately powered each wavelength group of RCLEDs, such that the light output duration for each wavelength was constant. The relative illumination timings are illustrated in the upper inset of Fig. 1, where the camera was triggered every time there was a switch of wavelength. The frame rate was set at $30 \mathrm{fps}$ ( $15 \mathrm{fps}$ for each wavelength) that was sufficient to recover the shape of the PPG arterial waveforms.
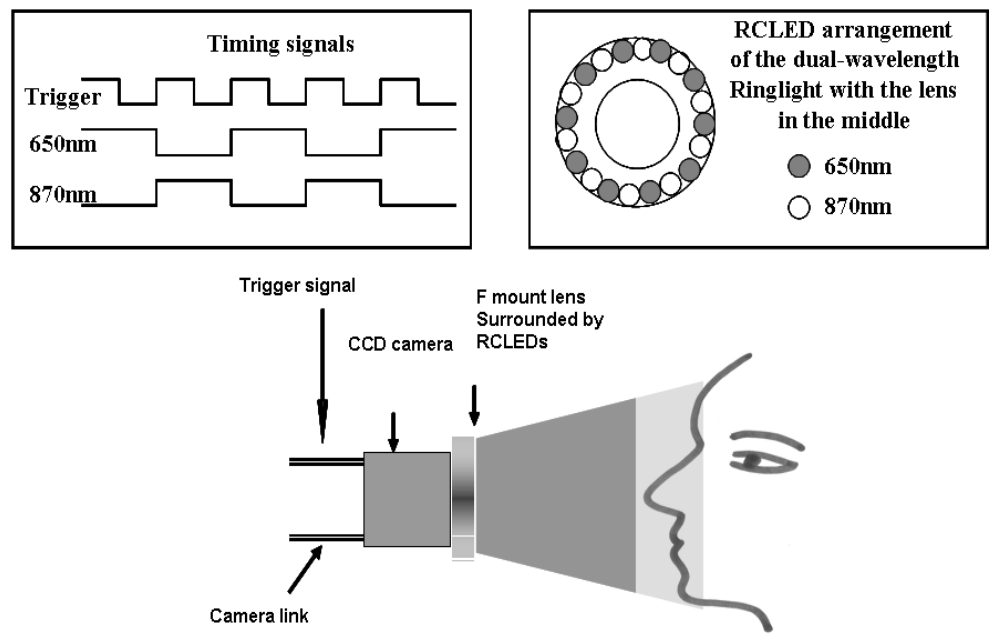

Fig. 1. Illustration of the remote PPG imaging system, depicting illumination and imaging geometry, relative timing signals, and RCLED arrangement of the dual-wavelength ring light (upper insets).

\subsection{Signal processing}

The captured frames were processed off-line in Labview (National Instruments Corp., USA) and Matlab (The MathWorks Inc., USA), and the 12-bit pixel values for each frame were saved as two Matlab 3-D matrices in the format of rows $\times$ columns $\times$ frame number, for each wavelength.

For the off-line processing, different regions of interest (ROI) can be chosen to investigate the spatial variation of blood perfusion variations. The average value of all pixels in each ROI box was tracked from frame to frame as depicted in Fig. 2. This process was performed for both frame sequences. 
After filtering-out noise and extraction of the DC component from the captured PPG waveform, a typical PPG AC pulsatile signal was derived in the time and frequency domains.

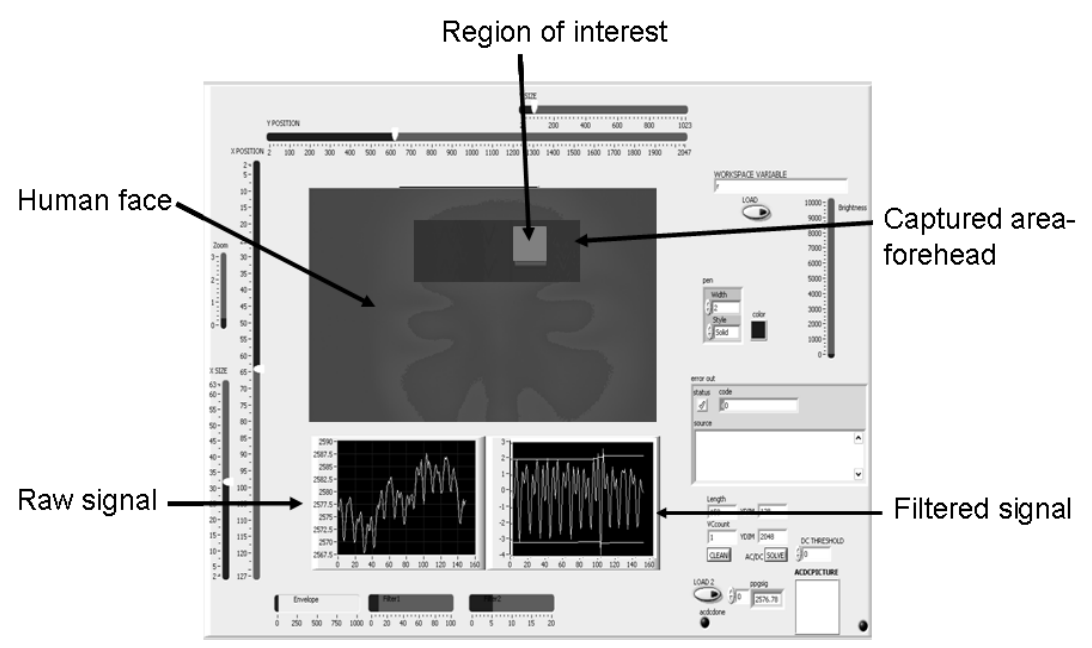

Fig. 2. Illustrated the processing window of imaging processing.

\subsection{Experimental protocol}

Eight healthy subjects ( 3 females, 5 males) participated in this study and the experimental protocol was performed in a dark room. The subjects were requested to seat in an upright position with their head resting on a bench with the eyes closed during the length of the experiment. A conventional transmission mode pulse oximetry probe (P861RA, ViaMed, UK) connected to a PPG board (DISCO4 PPG system, Dialog Devices Ltd, UK) was attached to the subjects' right index finger. The camera with the integrated ring light was positioned $13 \mathrm{~cm}$ from the face and focused on the subjects' forehead. The camera was configured to capture 300 frames at a resolution of $2048 \times 128$ pixels. A trigger signal was sent simultaneously to the camera system and the PPG board, initiating a 10s data acquisition in both systems.

\section{RESULTS AND DISCUSSION}

\subsection{Extraction of PPG waveforms}

The results of one subject from the remote PPG imaging system and conventional contact PPG system are presented in the time domain in Fig. 3 and in the frequency domain in Fig.4. It needs attention that signals correspond to the received light intensity rather than the absorbed light intensity, which would be directly proportional to the peripheral arterial pressure wave form. Referring to the signals from the remote imaging system, the systolic peaks and diastolic troughs are comparable to the contact PPG waveform and can adequately detect the dichrotic notch. The mean penetration depth for $650 \mathrm{~nm}$ is about $600 \mathrm{um}$ reaching to the papillary plexus layer and $1100 \mathrm{um}$ for $870 \mathrm{~nm}$ to the dermis layer ${ }^{[10,11]}$. Originated from the same heart beat, different shape and amplitude of the non-contact PPG pulsation signals are observed from the outputs illuminated by two different wavelength $650 \mathrm{~nm}$ and $870 \mathrm{~nm}$. These phenomena indicate the different penetration depths depending on the emitter wavelength, and also the distinct blood microcirculation at various vascular layers and areas in tissue. 

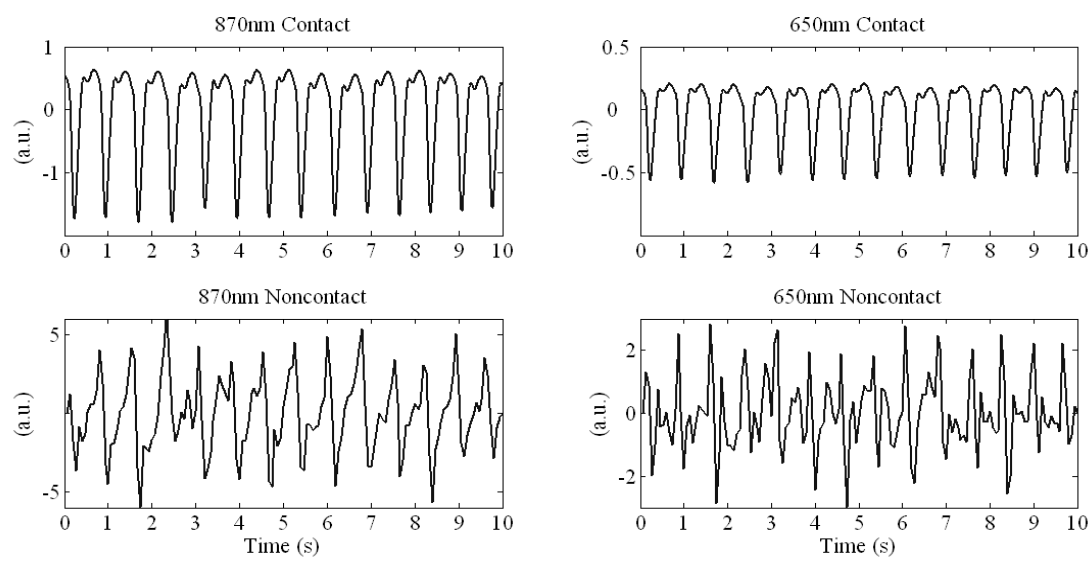

Fig. 3. The PPG signals from the conventional contact PPG imaging system and non-contact PPG system at $650 \mathrm{~nm}$ and $870 \mathrm{~nm}$ in time domain.
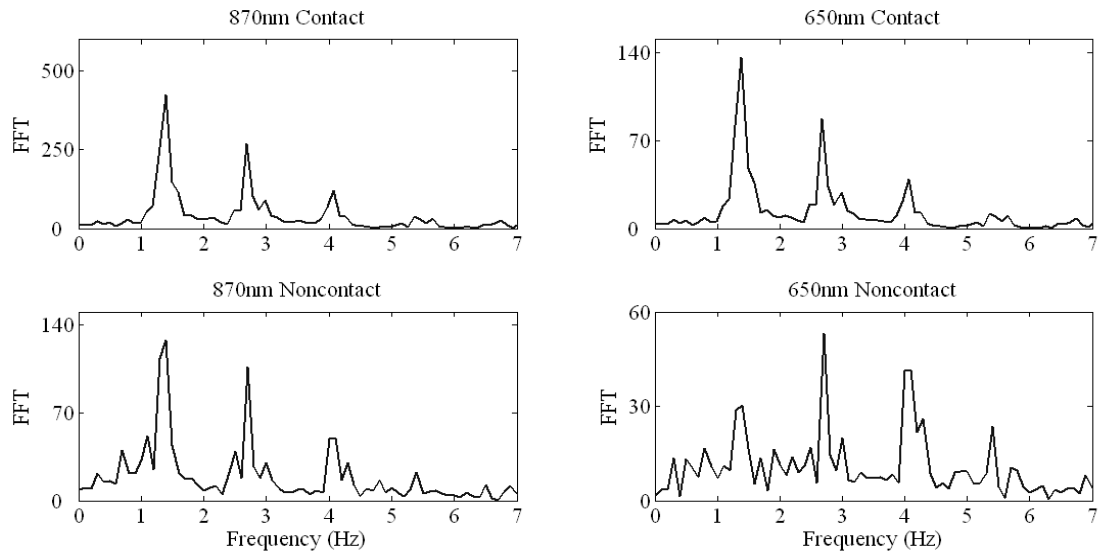

Fig. 4. The PPG signals from the conventional contact PPG imaging system and non-contact PPG system at $650 \mathrm{~nm}$ and $870 \mathrm{~nm}$ in frequency domain.

\subsection{Measurement of pulse rate in time domain}

In healthy subjects, the pulses of PPG waveform can provide an accurate measurement of heart rate. The pulse rate from the PPG imaging and contact PPG signals were compared to each other and a correlation coefficient was extracted to assess the level of agreement between the devices. Fig. 5 shows a scatter plot of the pulse rate measurement from the contact device versus the measurement from the non-contact device. The pulse rate was calculated from the mean duration $t_{p}$ of pulse-to-pulse intervals, i.e. pulse rate $=60 * 1 / t_{p}$, for the contact and non-contact PPG signals of each subject. The contact and non-contact $870 \mathrm{~nm}$ measurements are plotted as circles, and the $650 \mathrm{~nm}$ measurements as crosses. The regression line $y=0.9333 x+5.222$ is plotted in Fig. 5 , showing a high correlation $\left(r^{2}=0.9322\right)$ between the contact and non-contact devices, while a small offset is evident from the regression line equation. The non-contact method overestimates slightly the pulse rate. 


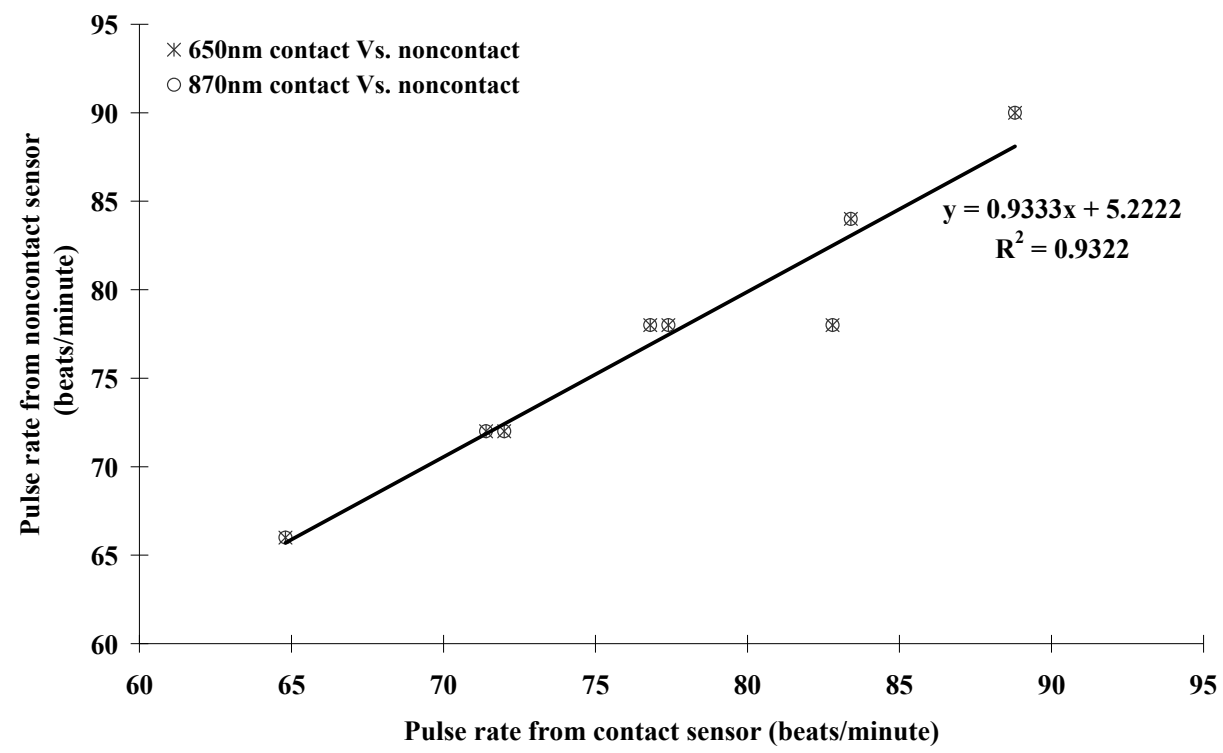

Fig. 5. Scatter plot showing the pulse rate from non-contact and contact measurement at $870 \mathrm{~nm}$ (circles) and $650 \mathrm{~nm}$ (crosses) for each subject. Also shown is the line $\mathrm{y}=0.9333 \mathrm{x}+5.222$, where both devices are highly correlated.

\subsection{Measurement of pulse rate in frequency domain}

Fig. 6 shows a Bland-Altman plot of the two methods, by comparing the frequency difference of cardiac component between PPG imaging and contact PPG, versus the mean frequency resulting from the two methods of measurement. Again data pertaining to $870 \mathrm{~nm}$ are represented by circles and $650 \mathrm{~nm}$ by crosses. The Bland-Altman plot ${ }^{[12]}$ is a popular method of assessing the level of agreement between a tested clinical device and a second device used as a gold standard.

A visual inspection of the plot enables us to identify the type and level of error by comparison to the true value of the variable of interest. The fundamental frequency of the cardiac cycle was extracted from the Fourier spectra separately for the contact and non-contact PPG signals. The mean frequency difference between the two techniques is $-0.00125 \mathrm{~Hz}$ and the standard deviation (SD) is $0.03336 \mathrm{~Hz}$. Assuming that the mean difference is normally distributed, the $95 \%$ confidence interval can be calculated as $\pm 1.96 \mathrm{SD}$, which means that pulse rate from PPG imaging might 3.8725 beats/minute above or 4.0226 beats/minute below the contact PPG. The insignificant mean difference means that the two methods can be used interchangeably for the measurement of pulse rate. 


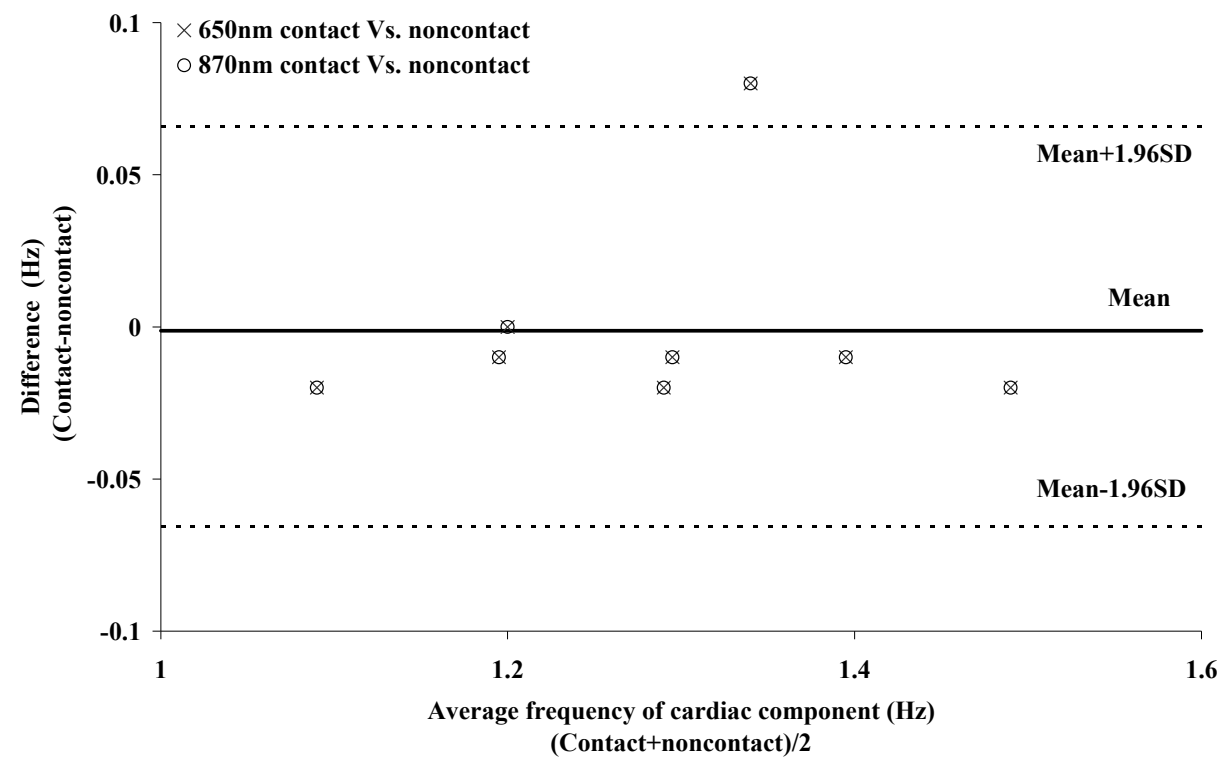

Fig. 6. Bland-Altman plot, showing the frequency difference of cardiac component between PPG imaging and contact PPG, versus the mean frequency resulting from the two methods of measurement for each subject. The circles indicate measurements at $870 \mathrm{~nm}$, and the crosses at $650 \mathrm{~nm}$. The solid line indicates the mean frequency difference and the dotted lines represent the \pm 1.96 standard deviations.

\section{CONCLUSIONS}

The PPG imaging technique presented in this paper performs comparably with a conventional contact device for pulse rate measurements and as a PPG waveform acquisition system. Although the camera is more susceptible to ambient light and motion artifact than a conventional contact probe, the potential of a non-contact imaging system to measure pulse rate and display PPG waveforms by non-contact means is only limited by the current configuration of the system. Such a device may not directly compete with conventional routine bedside monitoring devices or for ambulatory monitoring, but would most likely find applications in specialized areas such as wound surface diagnosis, arterial pulse transit time (PTT) measurement, or in conjunction with other modalities such as optical coherent tomography (OCT). Based on the capability of the PPG imaging to capture PPG waveforms using a multi-wavelength illuminator over a larger area, the 3$\mathrm{D}$ mapping of blood microcirculation by non-contact means is also in prospect.

With the introduction of the microelectronic programmable architectures, the above imaging PPG signal processing will be implemented by the means of a high-performance real-time signal processing system ${ }^{[13]}$ developed with a hybrid methodology of electronic system level (ESL) and register transfer level (RTL), targeted for intensive biomedical image processing to explicate physiological and pathological phenomena.

\section{ACKNOWLEDGEMENT}

The authors are grateful to all the members in the Photonics Engineering and Health Technology Research Group of Loughborough University for their enthusiastic support. Also, the authors would like to thank the financial support of Loughborough University (2006-2008). 


\section{REFERENCES}

[1] Kriss, T. C., Kriss, V. M., "History of the Operating Microscope: From Magnifying Glass to Microneurosurgery", Neurosurgery, 42 (4), 899-907 (1998).

[2] Hertzman, A. B., "Photoelectric plethysmograph of the fingers and toes in man," Proc. Soc. Exp. Biol. Med., 37, 529-534 (1937).

[3] Siegman, A. E. "Laser Beams and Resonators: The 1960s," IEEE J. Sel. Top. Quantum Electron., 6, 1380-1388 (2000).

[4] Brown, T. R., Goldstein, B., and Little, J., "Severe burns resulting from magnetic resonance imaging with cardiopulmonary monitoring. Risks and relevant safety precautions," Am. J. Phys. Med. Rehabil., 72 (3), 166-167 (1993).

[5] Wardell, K., Nilsson,G. E. “Duplex Laser Doppler Perfusion Imaging,” Microvasc. Res., 52, 171-182 (1996).

[6] Zheng, J., and Hu, S. "The preliminary investigation of imaging photoplethysmographic system," J. of Physics: Conference Series, 85, 012031(2007).

[7] Zheng, J., Hu, S. Azorin-Peris, V., Echiadis, A., Chouliaras, V., and Summers, R. "Remote simultaneous dual wavelength imaging photoplethysmography: a further step towards 3-D mapping of skin blood microcirculation," Proc. of SPIE, 6850, 68500S (2008).

[8] Wieringga, F. P., Mastik, F. and Van Der Steen, A. F. W., "Contactless Multiple Wavelength Photoplethysmographic Imaging: A First Step Toward 'SpO2 Camera' Technology,” Ann. Biomed. Eng., 33(8), 1034-1041 (2005).

[9] Humphreys K. and Ward, T., "Noncontact simultaneous dual wavelength photoplethysmography: A further step toward noncontact pulse oximetry," Rev. Sci. Instrum., 78, 044304 (2007).

[10] Kohen, E., Santus, R. and Hirschberg, J. G., Photobiology (Academic, 1995), p. 308

[11] Reuss, J. L., "Multilayer modeling of reflectance pulse oximetry," IEEE Trans. on Biomed. Eng. 52(2), 153-159 (2005).

[12] Altman, D.G., Bland, J. M. "Measurement in medicine: the analysis of method comparison studies," The Statistician, 32, 307-317 (1983).

[13] Chouliaras, V., Hu., S., Azorin-Peris, V., Echiadis, A., Summers, R., Zheng, J., King, I., "Real-time VLSI architecture for bio-medical monitoring", Technology and Applications in Biomedicine, 2008. ITAB 2008. International Conference, 463-466 (2008) 